\title{
POSSIBLE DETECTION OF BARYONIC FLUCTUATIONS IN THE LARGE-SCALE STRUCTURE POWER SPECTRUM
}

\author{
Christopher J. Miller AND RoBert C. Nichol \\ Department of Physics, Carnegie Mellon University, 5000 Forbes Avenue, Pittsburgh, PA-15232; chrism@cmu.edu, nichol@cmu.edu \\ AND \\ DAVID J. BATUSKI \\ Department of Physics and Astronomy, University of Maine, Orono, ME 04469; david.batuski@umit.maine.edu \\ Received 2001 January 8; accepted 2001 March 7
}

\begin{abstract}
We present a joint analysis of the power spectra of density fluctuations from three independent cosmological redshift surveys: the IRAS Point Source redshift catalog (PSCz) galaxy survey, the Automated Plate Measuring Machine (APM) galaxy cluster catalog, and the Abell/ACO cluster survey. Over the range $0.03 h \mathrm{Mpc}^{-1} \leq k \leq 0.15 h \mathrm{Mpc}^{-1}$, the amplitudes of these three power spectra are related through a simple linear-biasing model with $b=1.5$ and $b=3.6$ for Abell/ACO versus APM and Abell/ ACO versus PSCz, respectively. Furthermore, the shape of these power spectra are remarkably similar despite the fact that they are comprised of significantly different objects (individual galaxies through to rich clusters). Individually, each of these surveys shows visible evidence for "valleys" in their power spectra - i.e., departures from a smooth featureless spectrum - at similar wavenumbers. We use a newly developed statistical technique called the false discovery rate to show that these valleys are statistically significant. One favored cosmological explanation for such features in the power spectrum is the presence of a nonnegligible baryon fraction $\left(\Omega_{b}\right)$ in the universe, which causes acoustic oscillations in the transfer function of adiabatic inflationary models. We have performed a maximum likelihood marginalization over four important cosmological parameters of this model $\left(\Omega_{m}, \Omega_{b}, n_{s}\right.$, and $\left.H_{0}\right)$. We use a prior on $H_{0}=69 \pm 15$ and find $\Omega_{m} h^{2}=0.12_{-0.03}^{+0.02}, \Omega_{b} h^{2}=0.029_{-0.015}^{+0.011}$, and $n_{s}=1.08_{-0.20}^{+0.17}(2 \sigma$ confidence limits), which are fully consistent with the favored values of these cosmological parameters from the recent cosmic microwave background (CMB) experiments. This agreement strongly suggests that we have detected baryonic oscillations in the power spectrum of matter at a level expected from a cold dark matter model normalized to fit these CMB measurements.
\end{abstract}

Subject headings: cosmological parameters - galaxies: clusters: general — galaxies: general large-scale structure of universe - methods: statistical

On-line material: color figures

\section{INTRODUCTION}

We present a new analysis of the power spectra of density fluctuations $[P(k)]$ as derived from three recently available independent cosmological redshift surveys: the Abell/ACO cluster survey as defined in Miller \& Batuski (2001) and C. J. Miller et al. (2001a, in preparation), the IRAS Point Source redshift catalog (PSCz; Saunders et al. 2000), and the Automated Plate Measuring Machine (APM) galaxy cluster catalog (Dalton et al. 1994). For the first time, the volumes traced by these surveys are large enough to accurately probe the power spectrum to wavenumbers of $k=0.015$ (Abell/ACO), 0.025 (PSCz), and $0.030 h \mathrm{Mpc}^{-1}$ (APM). Throughout this discussion, we use $h=H_{0} / 100 \mathrm{~km}$ $\mathrm{s}^{-1} \mathrm{Mpc}^{-1}$.

Such information on the large-scale distribution of matter in the universe is critical for constraining cosmological models of structure formation as well as for determining the cosmological parameters. For example, the amplitude and shape of $P(k)$ below $k \sim 0.05 h \mathrm{Mpc}^{-1}$ can be used to discriminate between a high and low value of $\Omega_{m} h$, while a nonnegligible baryon fraction $\left(\Omega_{b}\right)$ would produce noticeable oscillations in $P(k)$ at $k<0.1 h \mathrm{Mpc}^{-1}$ (with the oscillations becoming broader and more easily detectable toward smaller $k$; see Eisenstein et al. 1998). Cosmological constraints based on the large-scale structure (LSS) in the universe are independent and complementary to those derived from Type Ia supernovae and cosmic microwave background (CMB) experiments (see Bond et al. 2001; Jaffe et al. 2000) and thus break key degeneracies inherent in cosmological measurements (see, e.g., Tegmark, Zaldarriaga, \& Hamilton 2001).

In the past, cosmological studies of the power spectrum of density fluctuations have been hampered in the following three ways: (1) uncertainties in the shape of $P(k)$ on very large scales, (2) the form of the relative biasing between the luminous and dark matter, and (3) the possible existence of a narrow "bump" in $P(k)$ (Landy et al. 1996; Einasto et al. 1997). As we will show in $\S \S 2$ and 3, our new data sets allow us to address these concerns and thus facilitate a more robust determination of the cosmological parameters from LSS measurements. Our work differs from other recent attempts to constrain cosmological models using LSS data (Novosyadlyj et al. 2000; Tegmark et al. 2001; Efstathiou \& Moody 2000; Huterer, Knox, \& Nichol 2001) since we first focus on the detection and interpretation of features in the matter power spectrum followed by a parameter estimation based on the favored models that explain these features.

\section{BIASING}

In Figure 1 (upper limits) we plot $P(k)$ for our three samples. The Abell/ACO power spectrum is from Miller \& Batuski (2001), the APM result is from Tadros, Efstathiou, 


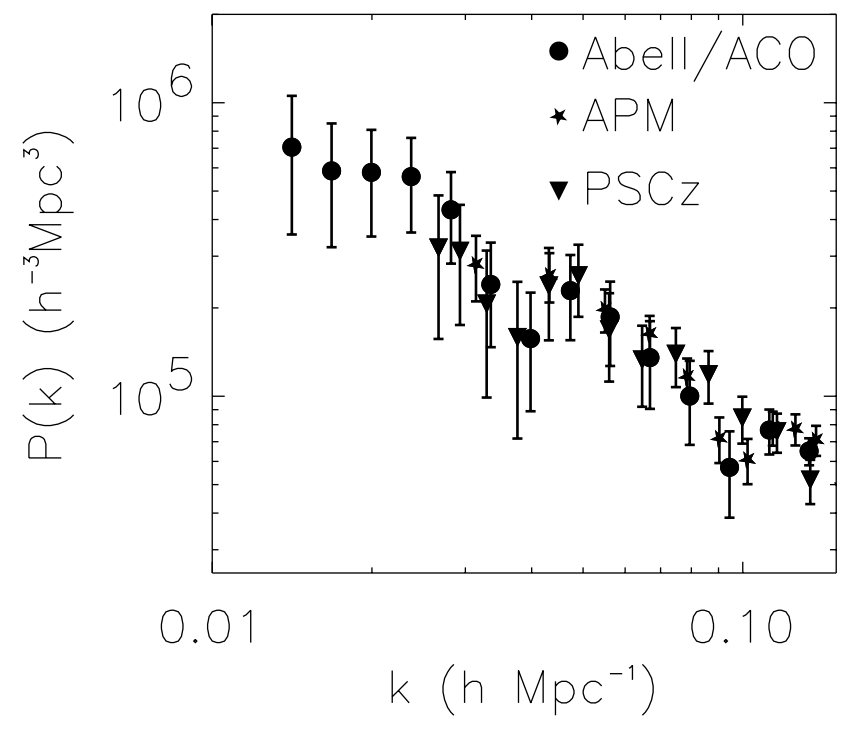

FIG. 1.-Power spectra for the three samples utilized in this work. The triangles are the PSCz galaxies, the stars are the APM groups and clusters, and the circles are the Abell/ACO rich clusters. The lower limts show $P(k)$ after a constant relative bias is applied to the data sets. The shift corresponds to $b=1.5$ and $b=3.6$ for Abell/ACO vs. APM and Abell/ACO vs. $\mathrm{PSCz}$, respectively. [See the electronic edition of the Journal for a color version of this figure.]

\& Dalton (1998), and the PSCz data are from Hamilton \& Tegmark (2000). In all three plots, we exclude any data with errors greater than $50 \%$ of the power for obvious reasons, but we note that their inclusion makes no difference in our final results. The errors are all $1 \sigma$ as quoted by the authors. In the lower limits, we plot the same data for the three samples after shifting the amplitudes of the APM and PSCz surveys to match those of the Abell/ACO survey. We have applied various techniques to calculate this amplitude shift (e.g., a $\chi^{2}$ minimization of the data with nearly identical $k$-values as well as using model fits to the data and renormalizing them to the Abell/ACO data), but in all cases, we obtain nearly identical results: the relative bias between the three samples over the range $0.03 h \mathrm{Mpc}^{-1} \leq k \leq 0.15 h$ $\mathrm{Mpc}^{-1}$ is $b=1.5$ and $b=3.6$ for Abell/ACO versus APM and Abell/ACO versus PSCz, respectively.

A remarkable aspect of Figure 1 is the overall success of a simple linear-biasing model in renormalizing the amplitudes of these power spectra over nearly a decade of scale. A scale-independent biasing model, over the scales discussed herein, has already been predicted from recent numerical simulations (Narayanan, Berlind, \& Weinberg 2000) and therefore allows us to confidently rescale these three different power spectra, thus facilitating the detection of features in the combined $P(k)$, as discussed below.

\section{THE SHAPE OF THE POWER SPECTRUM}

The overall shape of our combined power spectrum is shown in Figure 1, and it is unique for two reasons. First, we see no evidence at small $k$-values for a turnover in $P(k)$ toward a scale-invariant spectrum as previously hinted at in other LSS analyses (Tadros \& Efstathiou 1996; Peacock 1997; Gatzañaga \& Baugh 1998). This large-scale power also has been witnessed in other recently reported $P(k)$ measurements (see Efstathiou \& Moody 2000; Schuecker et al. 2001). Such large-scale power in $P(k)$ indicates a low value for the shape parameter $\left(\Gamma=\Omega_{m} h<0.3\right)$ since this has the effect of sliding the matter power spectrum to the smaller $k$-values compared to a critical matter density universe. Second, we do not find a narrow bump in $P(k)$ as reported by Einasto et al. (1997) and Landy et al. (1996) but instead witness "valleys" in the power spectrum. However, these previous surveys did not have the volume to see the largescale $(k<0.03)$ power in $P(k)$ and thus only saw the downturn of the valley, giving the appearance of a bump in the power spectrum at $k \simeq 0.05$, and therefore, our $P(k)$ may still be consistent with the Landy et al. and Einasto et al. power spectra. For the remainder of this section, we focus on the two valleys we see in Figure 1 at $k \simeq 0.035$ and $k \simeq 0.090 h \mathrm{Mpc}^{-1}$.

\subsection{False Discovery Rate}

In this section we investigate the statistical significance of the two features seen in Figures 1 and 2. We wish to determine whether all of the data points are consistent with being drawn from a smooth, featureless power spectrum. In the statistical literature, this is known as multiple hypothesis testing since one is testing, for each point, the null hypothesis that it was drawn from a featureless $P(k)$. The key issue then becomes choosing the threshold (in probability) at which these null hypotheses are rejected.

Traditionally, this is done by rejecting all points that are above a certain $\sigma_{\text {rej }}$ threshold. Unfortunately, there is a major problem with this methodology since the number of data points that are mistakenly rejected depends on the size of the data set. For example, if all our 37 data points were uncorrelated and truly drawn from a smooth $P(k)$, we would expect, on average, only 1.75 of these data points to be rejected (and thus in error) for a $\sigma_{\text {rej }}=2$ threshold. However, if we had $10^{6}$ data points, then the number of mistakenly rejected data points at the $\sigma_{\text {rej }}=2$ level would

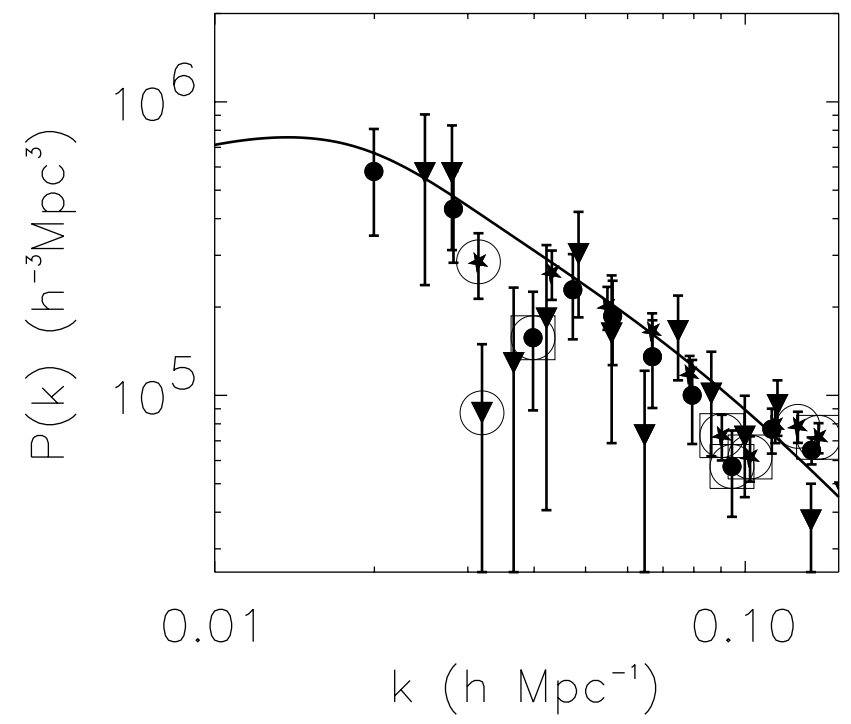

FIG. 2.-Amplitude-shifted power spectra for the three samples of uncorrelated data. The points highlighted with a circle denote rejections with $\alpha=0.25$ (e.g., one-quarter of the rejections may be mistakes). The points highlighted by squares are for $\alpha=0.11$ (e.g., $1 / 10$ of the rejections may be mistakes). The analysis utilizes our best-fit model with the baryon wiggles removed as the null hypothesis. By controlling the FDR, we can say with statistical confidence that the two valleys are detected as features in the power spectra. [See the electronic edition of the Journal for a color version of this figure.] 
be approximately 50,000 . (Note that this comparison only works if all of the real data were uncorrelated.) To guard against the overdetection of false discoveries, one could increase arbitrarily the significance threshold to $\sigma_{\text {rej }}=4$, thus reducing the number of errors, but that would lead to a much more conservative test. This is not to say that all of the $2 \sigma$ rejections would be wrong but simply that you would have many more false rejections. Thus, any significance threshold is arbitrary and highly dependent on the data size. So, enforcing a $\sigma_{\text {rej }}=3$ threshold for small data sets can be overly conservative. In summary, the more tests one does, the more stringent the required threshold becomes to avoid making too many false discoveries.

Ideally, we need a statistical technique that is more adaptive and whose interpretation does not depend on the data size. Instead of $\sigma_{\text {rej }}$, we will choose to control the false discovery rate $(\alpha)$-which is defined to be the percentage of mistakenly rejected points out of the total number of points rejected. This is clearly independent of the data size. Such an adaptive statistical tool is the false discovery rate (FDR; Benjamini \& Hochberg 1995). Once we choose $\alpha$, then the FDR procedure defines an appropriate significance threshold to obtain this false discovery rate for the data set in question. For example, if we choose $\alpha=\frac{1}{4}$ and reject eight data points, then, on average, only two of these points are in error even though their significance (as implied by their $\sigma$ ) may appear low. Again, arbitrarily setting $\sigma_{\text {rej }}=3$ for our data set may be too conservative. Instead, by a priori controlling the FDR, we can state with statistical confidence that six of eight rejected data points are true outliers against the null hypothesis. We briefly discuss the FDR here and refer the reader to Nichol et al. (2000) and C. J. Miller et al. (2001b, in preparation) for further details.

Operationally, we first compute the $p$-value ${ }^{1}$ for each test. Herein, we have used a smooth cold dark matter (CDM)

\footnotetext{
${ }^{1}$ The $p$-value is the probability that sampling from an ensemble of data sets would lead to a data value with an equal or higher deviation from the null hypothesis.
}

model based on our best-fit cosmological parameters (§ 4), but with the baryon oscillations removed (see Eisenstein \& $\mathrm{Hu}$ 1998). However, the reader should note that this model is nearly an exact power law over the range $k>0.02 h$ $\mathrm{Mpc}^{-1}$, and so our results would not change if we used a simple power law fitted to the data (without the valleys). We then rank, in increasing size, the $p$-values (for each test) and draw a line of slope $\alpha$ and zero intercept. Recall that $\alpha$ is the maximum acceptable FDR. The first crossing of this line with a $p$-value (moving from larger to smaller $p$-values) defines the significance threshold $\sigma_{\text {rej }}$ below which all points are rejected based on our null hypotheses. On average, only $\alpha$ times $100 \%$ of these rejected points will be in error.

Figure 2 presents the result of applying the FDR to our combined $P(k)$ using an uncorrelated data set (as opposed to the correlated data in Fig. 1). Specifically, we use the uncorrelated $P(k)$ given by Hamilton \& Tegmark (2000) for the PSCz, while Tadros et al. (1998) claim their APM $P(k)$ is uncorrelated; thus, we use their data points directly. For the Abell/ACO survey, Miller \& Batuski (2001) have shown that their $P(k)$ is uncorrelated for separations of $\Delta k \simeq 0.015$ $h \mathrm{Mpc}^{-1}$. Therefore, as can be seen from Figure 1, the data at $k>0.04$ are already uncorrelated, while for smaller $k$ values, we simply resample the data in such a way that the minimum separation between points is at least $\Delta k \sim 0.01 h$ $\mathrm{Mpc}^{-1}$. In Figure 2 the circled points are rejected (based on our null hypotheses) with an FDR of $\alpha=0.25$, while the points outlined with squares are rejected with an FDR of $\alpha=0.11$.

We detect the valleys at both $k \sim 0.035$ and $k \sim 0.09 h$ $\mathrm{Mpc}^{-1}$. The power of the FDR is that it ensures that no more than $25 \%$ of the eight rejections (circles) could be incorrect. If we apply the much more stringent constraint of $\alpha=0.11$, we reject five points (at $k \sim 0.09 h \mathrm{Mpc}^{-1}$ ), but the FDR limits the number of false rejections in this case essentially to zero. This allows us to state with statistical confidence that the fluctuations are true outliers against a smooth, featureless spectrum. Note that each of the three data sets contributes to the features, and so the detection is

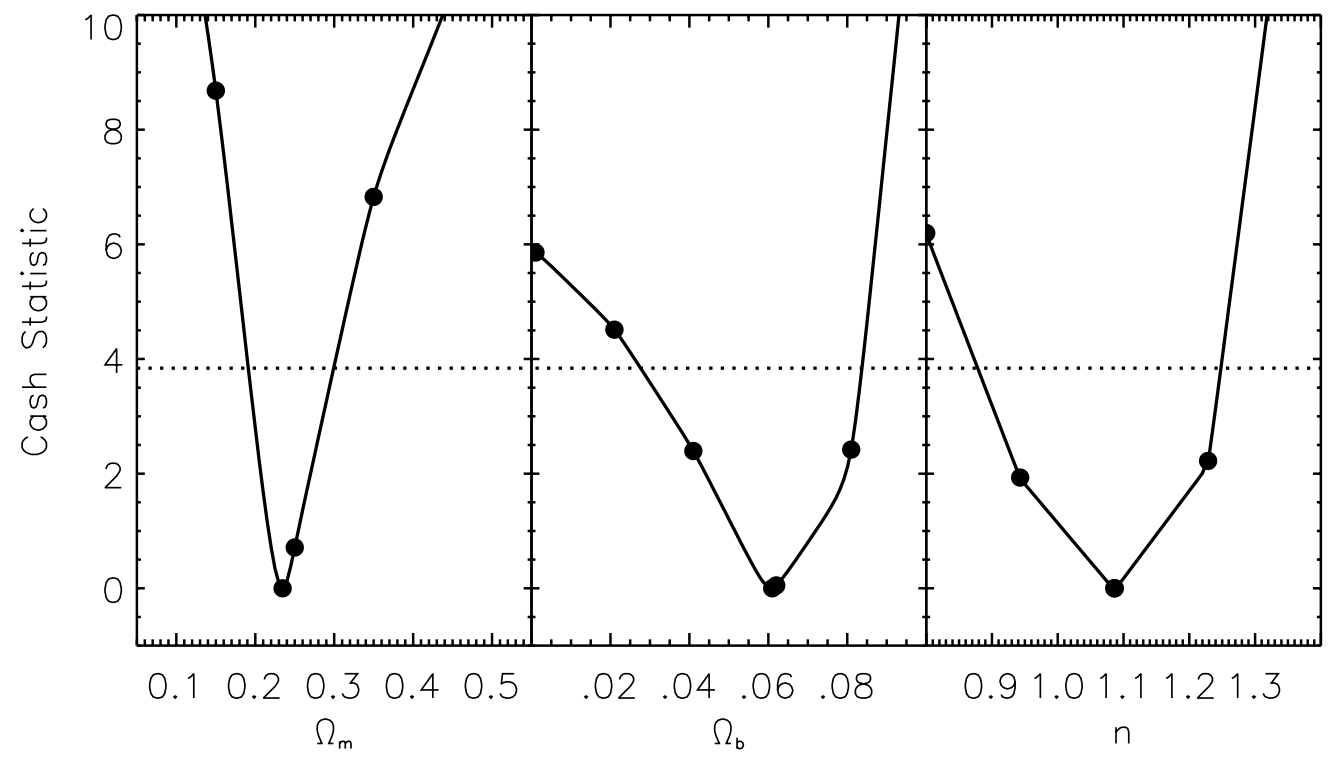

FIG. 3.-Cash statistic for the marginalized parameter estimations. The line corresponds to a $95 \%$ confidence region. [See the electronic edition of the Journal for a color version of this figure.] 
not dominated by one sample. In the next two sections, we review possible explanations for these observed features in our $P(k)$, including systematic uncertainties and cosmological effects.

\subsection{Systematic Uncertainties}

In this section we consider both measurement error and sampling effects as possible explanations for the features seen in the power spectra shown in Figures 1 and 2. To address the first of these, we simply note that Miller \& Batuski (2001) used several different methods of calculating the $P(k)$ for the Abell/ACO survey and observed no significant differences in the measured $P(k)$ for $k \leq 0.02 h \mathrm{Mpc}^{-1}$. Further evidence that the fluctuations seen in Figures 1 and 2 are not the result of the methodology comes from the fact that the authors of the three power spectra all used different methodologies to calculate their $P(k)$; the APM survey was analyzed using the method of Feldman, Kaiser, \& Peacock (1994), the analysis of the Abell/ACO survey followed Vogeley et al. (1992) and Feldman et al. (1994), while the PSC $\mathrm{P} P(k)$ was derived from a Karhunen-Loeve eigenmode analysis.

Next, we consider sampling effects: e.g., could artifacts of the design and construction of these surveys have produced such features and are the surveys independent and representative of the whole universe? We believe such effects are highly unlikely for two reasons. First, each of these three surveys was constructed in a different way and thus possesses significantly different window functions. For example, the APM survey only covers 1 sr of the sky centered on the southern Galactic cap with a near constant number density of clusters/groups over the redshift range $15,000 \mathrm{~km} \mathrm{~s}^{-1} \leq c z \leq 35,000 \mathrm{~km} \mathrm{~s}^{-1}$, while the Abell/ACO cluster sample covers over $2 \pi$ sr with a near constant number density of rich clusters out to $c z=42,000 \mathrm{~km} \mathrm{~s}^{-1}$ (in the north) and $c z=30,000 \mathrm{~km} \mathrm{~s}^{-1}$ (in the south). These two cluster surveys are independent of each other since $\simeq 90 \%$ of the APM clusters used by Tadros et al. (1998) are non-Abell systems and thus are not in the Abell/ACO sample (Miller \& Batuski 2001). In contrast, the PSCz survey covers $10.6 \mathrm{sr}(84 \%$ of the entire sky) and has a number density that falls off steeply beyond $c z=12,000 \mathrm{~km}$ $\mathrm{s}^{-1}$. Therefore, these three surveys sample different volumes of the universe, use different tracers of the mass (from galaxies through to rich clusters), and are independent of each other.

We stress here that these features are seen in all of the individual power spectra at similar wavenumbers and therefore are not a artifact of combining the three values of $P(k)$, which we did simply to increase the overall statistical significance of these valleys. This concordance is a powerful consistency check that argues against statistical and systematic uncertainties producing these features. Moreover, the volume sizes of these three surveys are so large that we hope to have reached a "fair sample" of the universe; thus these features cannot be explained away as unusual and as only present in our parts of the universe. We therefore believe that these fluctuations in $P(k)$ are physical, and in the next section we review possible cosmological explanations for them.

\subsection{Cosmological Explanations for the Fluctuations}

One possible cosmological explanation for these valleys in the observed $P(k)$ is the existence of corresponding fea- tures in the initial power spectrum of density fluctuations coming out of inflation. This explanation has been proposed for the excess power or correlations seen in several of the previous surveys (Broadhurst et al. 1990; Landy et al. 1996; Einasto et al. 1997). Unfortunately, the physical mechanism for producing such features in the initial power spectrum remains unclear (see Atrio-Barandela 2000; Einasto 2001).

A more natural and well-understood explanation is the presence of a nonnegligible baryon fraction in the universe, which leads to a coupling (at redshifts $z \geq 1000$ ) between the CMB photons and the baryonic matter, thus resulting in acoustic oscillations that leave an imprint on the matter power spectrum (see Eisenstein \& Hu 1998 and references therein). Recently, Eisenstein et al. (1998) examined this cosmological model and tested it against three LSS data sets: the APM deprojected $P(k)$ of Gaztañaga \& Baugh (1998), the $P(k)$ compilation of Peacock \& Dodds (1994), and $P(k)$ from the Abell/ACO sample from Einasto et al. (1997). Only the Einasto et al. $P(k)$ had a noticeable feature (bump) in the power spectrum, and Eisenstein et al. (1998) were unable to find a satisfactory cosmological model that fitted these data. Their analysis indicated two equally likely fits to the data, one with $\Omega_{m}<0.2$ and the other with $\Omega_{m}>$ 0.7 , while both models needed $\Omega_{b} / \Omega_{m} \simeq 0.3$. The high- $\Omega_{m}$ model was excluded by the big bang nucleosynthesis upper limit of $\Omega_{b} h^{2}<0.026$ (Burles, Nollett, \& Turner 2001), while the low- $\Omega_{m}$ model was rejected by the lack of largescale power seen in $P(k)$ for $k<0.05 h \mathrm{Mpc}^{-1}$.

We present here new constraints on model $P(k)$ using much improved data sets over those used by Eisenstein et al. (1998). The improvement in the data comes from the larger volumes traced by these surveys, thus allowing smaller values of $k$ to be probed with a higher resolution. In the next section, we reexamine this scenario and find that baryonic oscillations match with the power spectra in Figure 1. We note here that Tegmark et al. (2001) also

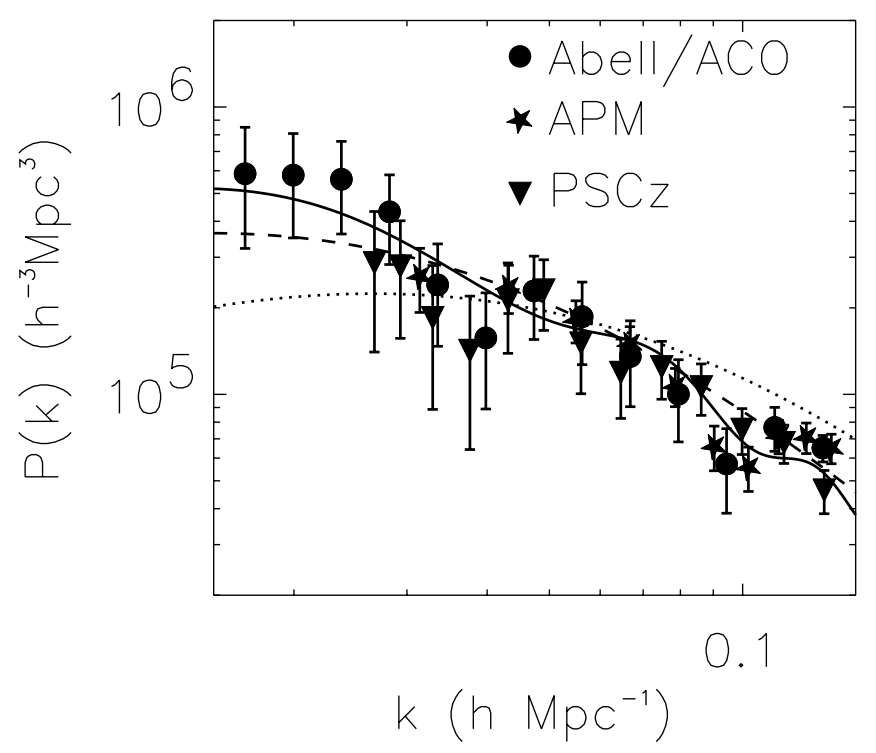

FIG. 4.-Best-fit model (solid line; $\Omega_{m}=0.24, \Omega_{b}=0.061$, and $n_{s}=1.08$ with $H_{0}=69$; see Table 1) plotted with the data from Fig. 1 (lower limits). The dotted and dashed lines are zero baryon $n_{s}=1$ models with $\Gamma=0.14$ and $\Gamma=0.25$, respectively. [See the electronic edition of the Journal for a color version of this figure.] 
hinted at the possible detection of baryon fluctuations in the PSCz $P(k)$, but a statistical analysis was not performed.

\section{COSMOLOGICAL PARAMETER ESTIMATION}

We have used the cosmological models of Eisenstein \& $\mathrm{Hu}$ (1998) to perform a parameter estimation that can be compared with the recent CMB results (Tegmark et al. 2001; Jaffe et al. 2000; Bond et al. 2001). We begin by constructing a four-dimensional grid in the parameter space using $\Omega_{m}, \Omega_{b}$, the spectral index $n_{s}$, and the Hubble constant. We apply a weak prior with $H_{0}=69 \pm 15 \mathrm{~km} \mathrm{~s}^{-1}$ $\mathrm{Mpc}^{-1}$, which is consistent and more conservative than the final results of the Hubble Space Telescope Key Project (Freedman et al. 2001). We then calculate the maximum likelihood via $\mathscr{L}=e^{-\chi^{2} / 2}$, where $\chi^{2}$ is calculated using the fitting formulae given in Eisenstein \& Hu (1998). In fitting these power spectra, we restrict ourselves to the range of $0.15 h \mathrm{Mpc}^{-1} \leq k \leq 0.15 h \mathrm{Mpc}^{-1}$ using the uncorrelated data of Figure 2.

The next issue is to find the global maximum in this multidimensional-likelihood space. Unfortunately, it is highly possible that this global maximum does not lie on one of our grid points, so to guard against this, we use the simplex method (see Press et al. 1992). We then marginalize over each parameter separately by fixing it to the grid and allowing the other three parameters to vary until we find the corresponding maximum. Our methodology is very similar to that of Tegmark \& Zaldarriaga (2000) except for our use of the simplex method to find the maximum likelihood. Tegmark \& Zaldarriaga fitted a cubic spline to their likelihood grid to find the maxima. However, this function can be ill-behaved if the surface is not smooth (i.e., if $\chi^{2}$ varies rapidly in the region of the minimum, which is often the case). Therefore, we advocate the use of the simplex method for future analyses since, in principle, it is less dependent on the actual likelihood surface. We note that a proper marginalization requires integration over the likelihood function. Tegmark et al. (2001) have shown that this is the same as the maximization technique used herein if the likelihood functions are Gaussian, which appears to be a reasonable approximation for our likelihood functions (see Fig. 3).

After marginalizing over the three power spectra separately, we combine the likelihoods together to arrive at our final results. For each of the samples, we allow the amplitude to be a free parameter. In this way, the bias parameter does not explicitly enter into the calculation. In Figure 3, we present the Cash statistic for each marginalized parameter (Cash 1979): $\operatorname{Cash}_{i}=-2 \ln \left(\mathscr{L}_{i} / \mathscr{L}_{\max }\right)$, where $\mathscr{L}$ is the maximum likelihood determined as a function of the fixed parameter $i$, and we allowed the other parameters to vary. $\mathscr{L}_{\text {max }}$ is the global maximum over all parameter space. If the log-likelihood functions can be well approximated with a second-order Taylor expansion, then the Cash statistic becomes analogous to a $\chi^{2}$ distribution. Thus, when we marginalize (i.e., hold one parameter fixed, allowing the others to vary), we have 1 degree of freedom and our $95 \%$ confidence limits are where our Cash statistic crosses a value of 3.84. In Table 1, we present our final estimates for the three cosmological parameters, $\Omega_{0}, \Omega_{b}$, and $n$. We also list similar recent results from the latest $\mathrm{CMB}$ data (Tegmark et al. 2001; Jaffe et al. 2000; Novosyadlyj et al. 2000). This table clearly illustrates that our best-fit values for these cosmological parameters are fully consistent with these other analyses.

In Figure 4, we present the data in Figure 1 along with our best-fit model shown in Table 1. For comparison, we also show two favored zero baryon $n_{s}=1$ models with $\Gamma=0.25$ and $\Gamma=0.14$. Clearly, the baryon model is the best representation given these three possibilities.

\section{CONCLUSIONS}

We present in this paper new evidence for the detection of statistically significant fluctuations in the matter power spectrum. The most natural explanation for these fluctuations are baryonic oscillations in a CDM universe as outlined in Eisenstein et al. (1998) and Eisenstein \& $\mathrm{Hu}$ (1998). Using this cosmological model, we have measured $\Omega_{m}, \Omega_{\mathrm{b}}$, and $n_{s}$, finding values that are fully consistent with those presently favored by the recent CMB experiments (see Table 1). This agreement is primarily due to the extra power seen on large scales (small $k$ ) as well as the features detected in all three power spectra. In the near future, surveys like the SDSS and 2dF Galaxy Redshift Survey (2dFGRS; Colless et al. 2000) will allow for a more detailed analysis of these baryonic features as well as provide more powerful constraints on the cosmological parameters than those presented here. However, we do note that on large scales, the volume sampled by the Abell/ACO survey discussed herein will remain unrivaled even after the main galaxy redshift surveys SDSS and 2dFGRS are completed and will thus remain, for some time, an important database for studying the large-scale structure in the universe. However, the SDSS Bright Red Galaxy (York et al. 2000) redshift survey will supersede all these surveys in terms of volume since it will provide a pseudo-volume-limited sample of galaxies out to $z \simeq 0.45$ carefully selected to sample the power spectrum of mass over as large a range of scales as possible.

We are in debt to Chris Genovese and Larry Wasserman for their help with FDR. We thank Christopher Cantaloupo, Adrian Melott, Wayne Hu, Peter Coles, Alex Szalay, Daniel Eisenstein, and Andrew Jaffe for helpful advise and suggestions throughout this work.

TABLE 1

Parameter Estimation Results

\begin{tabular}{|c|c|c|c|c|c|c|}
\hline$\Omega_{m} h$ & $\Omega_{b} h$ & $n_{s}$ & Confidence & Strong Priors & Data Used & Reference \\
\hline $0.12_{-0.03}^{+0.02}$ & $0.029_{-0.015}^{+0.011}$ & $1.08_{-0.20}^{+0.17}$ & $2 \sigma$ & & LSS & This work \\
\hline $0.22_{-0.06}^{+0.07}$ & $0.030_{-0.004}^{+0.004}$ & $0.99_{-0.06}^{+0.07}$ & $1 \sigma$ & $\Omega_{\text {tot }}=1$ & CMB & Jaffe et al. 2000 \\
\hline $0.15_{-0.15}^{+}$ & $0.054_{-0.03}^{+0.05}$ & $1.43_{-0.52}^{+}$ & $2 \sigma$ & & CMB & Tegmark et al. 2001 \\
\hline $0.23_{-0.12}^{+0.16}$ & $0.028_{-0.009}^{+0.008}$ & $0.96_{-0.10}^{+0.20}$ & $2 \sigma$ & & $\mathrm{CMB}+\mathrm{LSS}$ & Tegmark et al. 2001 \\
\hline $0.15_{-0.02}^{+0.04}$ & 0.02 & $0.92_{-0.09}^{+0.08}$ & $2 \sigma$ & $\Omega_{b}$ fixed & $\mathrm{CMB}+\mathrm{LSS}$ & Tegmark et al. 2001 \\
\hline
\end{tabular}




\section{REFERENCES}

Atrio-Barandela, F., Einasto, J., Müller, V., Mücket, J. P., \& Starobinsky, A. A. 2000, ApJL, submitted (astro-ph/0012320)

Benjamini, Y., \& Hochberg, Y. 1995, J. R. Soc. Stat. B, 57, 289

Bond, J. R., et al. 2001, in IAU Symp. 201, New Cosmological Data and the Values of Fundamental Parameters, ed. A. Lasenby, A. Wilkinson, \& A. W. Jones (San Francisco: ASP), 65

Broadhurst, T. J., Ellis, R. S., Koo, D. C., \& Szalay, A. S. 1990, Nature, 343, 726

Burles, S., Nollett, K. M., \& Turner, M. S. 2001, Phys. Rev. D, in press (astro-ph/0008495)

Cash, W. 1979, ApJ, 228, 939

Colless, M., et al. 2000, BAAS, 197, 89.05

Dalton, G. B., Croft, R. A. C., Efstathiou, G., Sutherland, W. J., Maddox, S. J., \& Davis, M. 1994, MNRAS, 271, L47

Efstathiou, G., \& Moody, S. J. 2000, preprint (astro-ph/0010478)

Einasto, J. 2001, in the Ninth Marcel Grossmann Meeting on General Relativity, ed. R. Ruffini (Singapore: World Scientific), in press (astro$\mathrm{ph} / 0011334)$

Einasto, J., et al. 1997, Nature, 385, 139

Eisenstein, D. J., \& Hu, W. 1998, ApJ, 496, 605

Eisenstein, D. J., Hu, W., Silk, J., \& Szalay, A. S. 1998, ApJ, 494, L1

Feldman, H. A., Kaiser, N., \& Peacock, J. A. 1994, ApJ, 426, 23

Freedman, W. L., et al. 2001, ApJ, 553, 47

Gatzañaga, E., \& Baugh, C. M. 1998, MNRAS, 294, 229

Hamilton, A. J. S., \& Tegmark, M. 2000, MNRAS, submitted (astro-ph/ 0008392)
Huterer, D., Knox, L., \& Nichol, R. C. 2001, ApJ, in press (astro-ph/ 0011069)

Jaffe, A. H., et al. 2000, Phys. Rev. Lett., 86, 3475

Landy, S. D., Shectman, S. A., Lin, H., Kirshner, R. P., Oemler, A. A., \& Tucker, D. 1996, ApJ, 456, L1

Miller, C. J., \& Batuski, D. J. 2001, ApJ, 551, 635

Narayanan, V. K., Berlind, A. A., \& Weinberg, D. H. 2000, ApJ, 528, 1

Nichol, R. C., et al. 2000, BAAS, 197, 107.03

Novosyadlyj, B., Durrer, R., Gottlober, S., Lukash, V. N., \& Apunevych, S. 2000, A\&A, 356, 418

Peacock, J. A. 1997, MNRAS, 284, 885

Peacock, J. A., \& Dodds, S. J. 1994, MNRAS, 267, 1020

Press, W. H., Teukolsky, S. A., Vetterling, W. T., \& Flannery, B. P. 1992, Numerical Recipes in C (2d ed.; Cambridge: Cambridge Univ. Press) Saunders, W., et al. 2000, MNRAS, 317, 55

Schuecker, $\mathrm{P}$. , et al. 2001, A\&A, in press (astro-ph/0012105)

Tadros, H., \& Efstathiou, G. 1996, MNRAS, 282, 1381

Tadros, H., Efstathiou, G., \& Dalton, G. 1998, MNRAS, 296, 995

Tegmark, M., \& Zaldarriaga, M. 2000, ApJ, 544, 30

Tegmark, M., Zaldarriaga, M., \& Hamilton, A. J. S. 2001, Phys. Rev. D, 63, 043007

Vogeley, M. S., Park, C., Geller, M. J., \& Huchra, J. P. 1992, ApJ, 391, L5

York, D. G., et al. 2000, AJ, 120, 1579 